\title{
FAR-INFRARED PROPERTIES OF MARKARIAN GALAXIES WITH MULTIPLE NUCLEI
}

\author{
J. M. Mazzarella and G. D. Bothun \\ University of Michigan \\ Department of Astronomy \\ Ann Arbor, MI 48109-1090
}

\section{Introduction}

We have coadded the IRAS data on a sample of 188 multiple-nucleus and morphologically peculiar Markarian galaxies noted as "interacting" in the compilation of Mazzarella and Balzano (1986). We have increased the number of objects in this sample with $60 \mu \mathrm{m}$ and $100 \mu \mathrm{m}$ IRAS detections by a factor of two from what is available in the Point Source Catalog, allowing the determination of farinfrared (FIR) luminosities and colors for over 130 morphologically peculiar Markarian galaxies. The galaxies in this sample have diameters less than $2^{\prime}$, and thus were seen as point sources by IRAS. All far-infrared properties discussed here apply to the entire multiple-nucleus or peculiar system. These data provide a unique opportunity to study the far-infrared properties of mergers which have prior indications of starburst or Seyfert activity as evidenced by their ultraviolet excess.

\section{Results and Discussion}

We present the first results of a search for correlations between the far-infrared luminosities and colors of these galaxies with various optical properties of the systems, including the projected separation and relative velocity of the nuclei, emission line properties, and reddening as measured from optical long-slit spectra of approximately 60 systems.

\subsection{FAR-INFRARED COLORS}

A histogram of the ratio $f_{60} / f_{100}$, as determined from the coadded IRAS data for 149 objects detected in these two bands, indicates that $60 \%$ of the systems have $f_{60} / f_{100}>0.5$. The dominance of the warm dust component in these systems provides evidence that the multiple-nucleus Markarian galaxies are predominantly closely interacting galaxy pairs and ongoing mergers.

The large number of sources detected at $25 \mu \mathrm{m}, 60 \mu \mathrm{m}$, and $100 \mu \mathrm{m}$ makes this sample extremely useful for examining the relative frequency of AGNs with respect to starburst nuclei in this sample, as well as investigating the efficiency of far-infrared color criteria versus optical/ultraviolet techniques for distinguishing Seyfert galaxies from the general galaxy population. Analysis of a color-color diagram for all Markarian galaxies with currently available $I R A S$ detections at $25 \mu \mathrm{m}, 60 \mu \mathrm{m}$, and $100 \mu \mathrm{m}$ (152 from the Point Source Catalog (PSC) and 85 ADDSCAN detections from the present study) reveals the following facts. A lack of space precludes including the plots. 
1) $29 \%$ of the 85 interacting/merging systems are contained in the region proposed to preferentially contain Seyfert galaxies (Miley et al. 1985), with a tolerance of \pm 0.2 in either color, compared to $28 \%$ of the 152 PSC sources (which excludes the multiple-nucleus systems). There is no significant excess of Seyfert galaxies in the multiple-nucleus sample with respect to the general Markarian sample that excludes the mergers. AGN classifications provided by optical spectroscopy also support this result in that only $9 \%$ of the galaxies in the multiple-nucleus/peculiar sample contain at least one Seyfert or LINER nucleus, a result which is very close to the relative ratio of AGNs to $H$ II-type galaxies in the general Markarian sample. These results imply strongly that although starburst and Seyfert nuclei are common among interacting/merging systems, strong interactions or mergers do not favor the production of Seyfert nuclei over nuclear starbursts.

2) A substantial number of Markarian Seyferts are not located in the expected region of the color-color diagram. The "Seyfert zone" misses $30 \%$ of the known optically classified AGNs in the Markarian sample (Mazzarella and Balzano 1986). This concurs with the finding of Salzer and MacAlpine (1988) for optically selected AGNs in the UM survey. Moreover, a larger percentage of optically-selected Seyfert $2 \mathrm{~s}$ are located outside of the "Seyfert" zone than Seyfert 1s. A similar situation is seen in the data of Bothun et al. (1988) and Salzer and Macalpine. Clearly both optical and far-infrared selection techniques must be employed to obtain a complete sample of AGNs.

\subsection{CORRELATIONS WITH OPTICAL EMISSION-LINE PROPERTIES}

Long-slit spectroscopic observations have been obtained for sixty multiple-nucleus Markarian galaxies which have IRAS detections in the present study (Mazzarella and Boroson 1989). Preliminary results indicate no significant correlations between $\Delta(\mathrm{cz})$ or projected separation of the nuclei with any FIR or optical emission-line properties examined so far. However, two significant correlations have been found. The mean reddening parameter of the nuclei/knots in a given system, $c(H \beta)$, as determined from $H \alpha / H \beta$, seems to correlate with the global infrared excess, $\log \left(L_{F I R} / L_{B}\right)$, in the sense that large optical nuclear reddening corresponds to large $L_{F I R} / L_{B}$. A linear correlation coefficient of 0.48 with 50 data points indicates a probability of less than 0.001 that these variables are linearly uncorrelated. Similarly, the Spearman rank correlation statistic indicates a $3 \sigma$ correlation with a probability of $0.3 \%$ of being due to chance. Salzer and MacAlpine find no such relation in the UM galaxy sample, and they discuss at length why such a correlation may be "washed out" in their sample. We also have evidence for a statistically significant relation between the the diagnostic line flux ratio $[\mathrm{O} \mathrm{III}] \lambda 5007 / \mathrm{H} \beta$ and $f_{60} / f_{100}$, in the sense that higher ionization correlates with hotter dust temperature. A linear correlation coefficient of 0.50 with 45 data points indicates a confidence level of 3.3 $\sigma$. These results suggest that something is clearly unique about this sample. Correlations between nuclear emission-line properties and FIR emission as measured by IRAS suggest that the FIR emission is dominated by the hot nuclear components of these galaxies.

We would like to thank the helpful staff of the Infrared Processing and Analysis Center, particularly E. Smith and W. Rice, for their assistance during the visits of J. M. M. This research was supported by NASA through JPL Contract Number 957979.

\section{References}

Bothun, G., Halpern, J., Lonsdale, C., Impey, C., and Schmitz, M., 1988, preprint. Miley, G. K., Neugebauer, G., and Soifer, B. T. 1985, Ap. J. (Letters), 293, L11. Mazzarella, J. M., and Balzano, V. A. 1986, Ap. J. Suppl., 62, 751.

Mazzarella, J. M., and Boroson, T. A. 1989, in preparation.

Salzer, J. J., and MacAlpine, G. M. 1988, A. J., in press. 